\title{
Retraction Note to: Financial information prediction and information sharing supervision based on trend assessment and neural network
}

\author{
Xingyu Gao ${ }^{1} \cdot \mathrm{Pu}$ Zhang $^{2} \cdot$ Guanhua Huang $^{3} \cdot$ Hui Jiang ${ }^{4} \cdot$ Zhuo Zhang $^{5}$
}

Published online: 24 February 2022

(C) Springer-Verlag GmbH Germany, part of Springer Nature 2022

\section{Correction to: Soft Computing (2020) 24:8087-8096 https://doi.org/10.1007/s00500-019-04176-z}

The authors have retracted this article because after its publication, they noticed that there were problems with the data source. The paper adopted the method of using 'the first successfully matched table' for the data extraction which is correct in cases where the consolidated statement comes first and the parent company statement comes second. However, using this method some of the financial data used came from the parent company while some of the data came from consolidated statements. This caused errors in the initial sample set and so the conclusions made in this article can no longer be considered accurate. All authors agree to this retraction.

Publisher's Note Springer Nature remains neutral with regard to jurisdictional claims in published maps and institutional affiliations.

The original article can be found online at https:// doi.org/10.1007/s00500-019-04176-z.

Zhuo Zhang

$876057891 @ q q . c o m$

Xingyu Gao

gxy116@mail.sdu.edu.cn

Pu Zhang

zhangpu85@126.com

Guanhua Huang

drbrucehuang@hotmail.com

Hui Jiang

1016610374@qq.com

1 School of Business, Shandong University, Weihai, China

2 Planning and Development Office, China Development Bank Hebei Branch, Shijiazhuang, China

3 Business School, University of International Business and Economics, Beijing, China

4 School of Economics and Management, Shanghai Maritime University, Shanghai, China

5 School of Business, Macau University of Science and Technology, Taipa, Macau S.A.R., China 